\title{
Cohesión, adaptabilidad y composición familiar en adolescentes del Callao, Perú
}

\section{Family Cohesion, Adaptability and Composition in Adolescents from Callao, Peru}

\author{
David Villarreal-Zegarra* \& Angel Paz-Jesús \\ Instituto Peruano de Orientación Psicológica, Lima, Perú.
}

Recibido 28-04-17

Aprobado 31-07-17

En Línea 10-08-17

*Correspondencia

Email: davidvillarreal@ipops.pe
Citar como:

Villarreal-Zegarra, D., \& Paz-Jesús, A. (2017).

Cohesión, adaptabilidad y composición familiar en adolescentes del Callao, Perú. Propósitos y Representaciones, 5(2), 21 - 64. doi: http://dx.doi.org/10.20511/pyr2017.v5n2.158

(c) Universidad San Ignacio de Loyola, Vicerrectorado de Investigación, 2017.

(cc) BY-NC-ND Este artículo se distribuye bajo licencia CC BY-NC-ND 4.0 Internacional (http://creativecommons.org/licenses/by-nc-nd/4.0/). 


\section{Resumen}

El objetivo del estudio consistió en analizar si existen diferencias significativas entre la cohesión y adaptabilidad familiar según el tipo de composición familiar (nuclear, extensa, monoparental y anuclear). Esta es una investigación no experimental, de tipo empírica, en la que se empleó una estrategia asociativa selectiva no probabilística y transversal; se evaluó a 428 adolescentes varones y mujeres, entre 14 a 18 años, estudiantes en un colegio nacional del Callao. Se utilizó la escala de evaluación de la cohesión y la adaptabilidad familiar de Olson (FACES III) y un cuestionario ad hoc para evaluar la composición familiar. Los resultados muestran diferencias significativas entre los adolescentes pertenecientes a familias nucleares y anucleares. Se discuten implicaciones teóricas y prácticas.

Palabras clave: Cohesión familiar, adaptabilidad familiar, composición familiar, adolescentes, modelo circumplejo, análisis factorial.

\section{Summary}

The objective of the study was to analyze if there are significant differences between family cohesion and adaptability according to the type of family composition (nuclear, extended, single parent and non-nuclear). This is a non-experimental and empirical research, in which a non- probabilistic, cross-sectional, selective and associative strategy was employed; 428 male and female teenagers, aged 14 to 18 , from a public school in Callao were evaluated. The D. Olson's family cohesion and adaptability evaluation scale (FACES III) and an ad hoc questionnaire were used to evaluate family composition. The results show significant differences between adolescents belonging to nuclear families and adolescents belonging to non-nuclear families. Theoretical and practical implications are discussed.

Keywords: Family cohesion; family adaptability; family composition; adolescents; circumplex model, factor analysis. 


\section{Introducción}

La formación educativa ha mostrado la importancia de la relación escuelafamilia para que este proceso sea exitoso (Martínez, 2010), específicamente en el caso de los adolescentes, el apoyo familiar constituye un factor protector importante (Orcasita \& Uribe, 2010). En este sentido, los estudios han descrito las diferentes representaciones de los adolescentes, con base en la percepción que tienen sobre la forma en que se relacionan en su hogar (Scarpati, Pertuz \& Silva, 2014). No obstante, la complejidad de la dinámica familiar, ha representado un reto para los investigadores en las ciencias sociales (López-Montaño \& Herrera-Saray, 2014).

El mapa mundial de la familia (Child Trends, 2013) plantea indicadores que permiten analizar la complejidad de las relaciones familiares mediante la estructura familiar, nivel socioeconómico familiar, procesos familiares y cultura familiar. Asimismo ha permitido identificar variaciones contemporáneas respecto a la dinámica familiar en los diferentes países de América. Estas variaciones se traducen en las nuevas formas que encuentran los miembros de la familia para vincularse, establecer límites/reglas, comunicarse entre sí y unirse en nuevos sistemas familiares o composición familiar

Las características en la composición familiar permiten esquematizar la estructura que enmarca las interacciones entre los miembros del hogar (Suárez et al., 2015). No obstante, existen diferentes formas de composición familiar (ej: familias nucleares, monoparentales, extensas y anucleares), algunas de ellas pueden generar una respuesta social negativa, especialmente en contextos conservadores como el latinoamericano (Faúndes, 2012). Esto resulta crucial debido a la susceptibilidad del adolescente a la crítica social, por lo que repercute significativamente en el grado de funcionalidad que perciba en su familia (Cervini, Dari \& Quiroz, 2016).

Asimismo, los tipos de composición familiar influyen en los niveles de inestabilidad emocional; la composición nuclear se ha asociado con un nivel 
bajo de inestabilidad emocional, mientras que una composición extensa o familia extensa se vincula a un nivel mayor de inestabilidad emocional (Bardales, 2005).

La funcionalidad familiar es definida como la efectividad de la familia para conseguir cierto equilibrio, orden y unidad ante las exigencias del ciclo vital (Staccini, Tomba, Grandi, \& Keitner, 2015). Respecto a las dimensiones centrales de la funcionalidad familiar, la propuesta de Olson et al. (1982), señala la cohesión, adaptabilidad, comunicación y satisfacción familiar (Copez-Lonzoy, Villarreal-Zegarra, \& Paz-Jesús, 2016; Olson, 2011). Asimismo, Olson (2000) plantea que la funcionalidad familiar se rige principalmente por la capacidad de adaptación y grado de cercanía emocional entre los miembros.

En este sentido la composición y la funcionalidad familiar representan dos variables cuya relación resulta un tema controversial en la actualidad (Scarpati, Pertuz, \& Silva, 2014). Si bien es cierto, desde la perspectiva teórica se establece que no se trata del tipo de familia y la relación por parentesco sanguíneo, biológico, cultural, comunitario o legal; sino de las características de vinculación entre sus miembros (Bermúdez \& Brik, 2010), aún es necesario mayor evidencia empírica al respecto.

Las investigaciones sobre la influencia de la familia en el desarrollo psicosocial del adolescente aún resultan escasas a nivel nacional; no obstante se reconoce su importancia para comprender y delimitar respuestas en diversos problemáticas psicosociales. Es por ello que este estudio busca conocer la relación entre la composición y la funcionalidad familiar en adolescentes de la provincia del Callao (Perú). Además se pretende recabar evidencia psicométrica sobre la escala FACES III. 


\section{Método}

\section{Diseño.}

La presente es una investigación no experimental, de tipo empírica que emplea una estrategia asociativa selectiva no probabilística y trasversal (Ato, López \& Benavente, 2013). Asimismo no se utilizan variables manipuladas y se busca describir una realidad en un momento determinado, a la vez que se hacen comparaciones entre grupos.

\section{Participantes.}

La muestra está conformada por adolescentes $(n=428)$ varones $(n=242$, $56.5 \%$ ) y mujeres $(n=186,43.5 \%)$ de quinto año de secundaria de un colegio nacional del Callao (Perú). Las edades están en el rango de 14 a 18 años, la edad promedio es de 16 años y 3 meses. Sobre la composición familiar se encuentra la familia nuclear $(n=170,39.7 \%)$, monoparental $(n=56,13.1 \%)$, extensa $(n=166,38.8 \%)$ y anuclear $(n=36,8.4 \%)$.

Según el número de hermanos, los participantes pueden ser hijos únicos ( $n=15,3.5 \%)$, con un hermano $(n=124,29 \%)$, dos hermanos $(n=134,31.3)$, tres hermanos $(n=93,22 \%)$ o con cuatro a más hermanos $(n=56,14.2 \%)$.

\section{Instrumentos.}

Escala de evaluación de la cohesión y la adaptabilidad Familiar de D. Olson - FACES III (1982).

Está conformada por 20 ítems, la confiabilidad de la prueba original es de .68, y según sus dimensiones, cohesión .77 y adaptabilidad .62. Su corrección implica la suma de los ítems impares para la dimensión de cohesión, y de los ítems pares para de adaptabilidad; según la traducción utilizada por Reusche (1998). Esta escala presenta dos dimensiones y, con base en los puntajes, permite esquematizar cuatro tipos de familia. 
Cohesión familiar: Esta dimensión evalúa el grado en que los miembros de la familia están separados o conectados entre sí y son capaces de apoyarse unos a otros. Se define como el vínculo emocional entre los miembros. De esta manera los niveles de cohesión oscilan entre: Desligada (D), Separada (S), Conectada (C) y Aglutinada (A). Donde uno de los ítems más representativos es: "Los miembros de la familia se sienten muy cerca uno del otro".

Adaptabilidad familiar: Esta dimensión examina la habilidad del sistema marital o familiar para cambiar su estructura de poder, sus roles y reglas de relación en respuesta a una demanda situacional o de desarrollo. Los niveles de adaptabilidad dan lugar a los niveles: Rígida (R), Estructurada (E), Flexible (F), Caótica (C). (Olson et al., 1982). Donde uno de los ítems más representativos es: "Las reglas cambian en nuestra familia".

A través de la correlación curvilínea de estas dos dimensiones, se determinan tres niveles de funcionalidad familiar: familias balanceadas, extremas y de rango medio.

La composición familiar hace referencia al número de miembros y el parentesco sanguíneo, legal o afectivo que puedan tener. Se clasifica en familia nuclear (ambos padres biológicos/legales que viven junto a sus hijos biológicos/legales), familia extensa (uno o ambos padres biológicos/ legales que viven junto a sus hijos biológicos/legales y otros miembros como abuelos, tíos, primos, etc.), familia monoparental (sólo uno de los padres biológicos/legales que viven junto a sus hijos biológico/legales) y familia anuclear (menores de edad que viven sin sus padres biológicos/legales, otros miembros como abuelos, tíos, primos o hermanos son los que cumplen las funciones parentales).

Para clasificar a los adolescentes según su composición familiar, se desarrolló una ficha ad hoc la cual señala con cuales miembros de la familia vive el menor (madre, padre, hermanos, tíos, abuelos y primos) y adicionalmente registra la edad, sexo, grado de instrucción, sección, número 
de hermanos, lugar que ocupa entre los hermanos y con quienes vive actualmente.

\section{Procedimiento.}

El inventario se administró en las aulas de clase de la institución educativa y en un ambiente ventilado y limpio, esto a fin de contar con las condiciones propicias para la evaluación. Antes de aplicar las pruebas se explicó el propósito de la evaluación, se dieron las indicaciones respectivas y se suplió las dudas de los participantes. Cabe señalar que ocho de las pruebas fueron anuladas debido a estar incompletas.

En el análisis de los datos se utilizaron estadísticos descriptivos (tendencia central, dispersión, curtosis y asimetría), estadísticos de consistencia interna (Alfa de Cronbach, alfa ordinal, omega), análisis factorial exploratorio y confirmatorio, estadísticos para analizar la normalidad con la prueba de Shapiro y Wilk (1965; Dominguez-Lara, 2016; Ghasemi, \& Zahedias1, 2012), para en análisis inferencial de diferencia de medias ( $h$ de Kruskal-Wallis) y para la asociación (Chi-cuadrado de Pearson, tablas de contingencia y la V de Cramer).

\section{Resultados}

A nivel descriptivo se analizaron las medidas de tendencia central, de dispersión y normalidad en las dimensiones de cohesión y adaptabilidad, con el fin de seleccionar los estadísticos más apropiados a la naturaleza de los datos. 


\section{Tabla 1.}

Medidas de tendencia central y de dispersión de las dimensiones de cohesión y adaptabilidad familiar.

\begin{tabular}{llllllll}
\hline & $M$ & $D E$ & $S W$ & $A s$ & $K s$ & Min & Max \\
\hline Cohesión & 33.95 & 6.51 & $.964(p<.001)$ & -.714 & 1.136 & 12 & 48 \\
\hline Adaptabilidad & 26.90 & 5.03 & $.989(p=.002)$ & -.006 & .227 & 13 & 42 \\
\hline
\end{tabular}

Nota: Los puntajes para cada dimensión pueden ir entre 10 a 50.

Los primeros hallazgos muestran que los datos no se ajustan a la curva normal (Ghasemi \& Zahediasl, 2012), por lo que se determina el uso de estadísticos no paramétricos. Asimismo, se analizó la frecuencia y porcentaje de los tipos de cohesión, adaptabilidad y funcionalidad. Se dispuso de los puntos de cohorte el baremo para Lima Metropolitana de Toscano (1998), mediante el cual se agrupó los tipos de cohesión y adaptabilidad según el puntaje directo con el fin de conocer la prevalencia sobre los tipos de dinámica familiar.

\section{Tabla 2.}

Medidas de frecuencia y porcentaje de los tipos de cohesión, adaptabilidad $y$ funcionalidad familiar $(n=428)$.

\begin{tabular}{llll}
\hline & & $f$ & $\%$ \\
\hline Tipo de cohesión & Dispersa $^{\mathrm{e}}$ & 52 & 12.1 \\
& Separada $^{\mathrm{b}}$ & 128 & 29.9 \\
& Conectada $^{\mathrm{b}}$ & 190 & 44.4 \\
& Aglutinada $^{\mathrm{e}}$ & 58 & 13.6 \\
\multirow{2}{*}{ Tipo de adaptabilidad } & Rígida $^{\mathrm{e}}$ & 118 & 27.6 \\
& Estructurada $^{\mathrm{b}}$ & 168 & 39.3 \\
& Flexible $^{\mathrm{b}}$ & 118 & 27.6 \\
& Caótica $^{\mathrm{e}}$ & 24 & 5.6 \\
\hline
\end{tabular}

Nota: ${ }^{b}=$ corresponde a tipos balanceados; ${ }^{e}=$ corresponde a tipos extremos 


\section{Análisis de la estructura interna.}

Se optó por dividir la muestra en dos grupos equivalentes de 214 adolescentes cada uno a fin de realizar el análisis factorial exploratorio (AFE) y el análisis factorial confirmatorio (AFC). En el análisis previo a la aplicación del AFE se analizó la medida de adecuación muestral de Kaiser-Meyer-Olkin (KMO); donde medidas mayores a .80 se consideran óptimas, y valores menores .70 como mediocres (Pardo \& Ruiz, 2002; Hair, Anderson, Tatham \& Black, 1999). Asimismo la prueba de Bartlett también presentó niveles adecuados en ambos casos e indicó suficiente intercorrelación de los ítems, por lo cual resultó coherente la aplicación del análisis factorial, en solo los datos de la dimensión de cohesión. Al analizar la varianza explicada, sólo la dimensión de cohesión mostró un valor mayor de $40 \%$; por lo que fue la única con valor adecuado (Lloret-Segura, Ferreres-Traver, Hernández-Baeza \& TomásMarco, 2014).

Se utilizaron matrices policóricas conforme a la naturaleza de las variables (discretas o no continuas) (Domínguez, Villegas \& Centeno, 2014) (ver tabla 2).

\section{Tabla 3.}

Análisis de los supuestos mínimos para realizar análisis factorial exploratorio.

\begin{tabular}{lccll}
\hline & & & & \\
& Determinante & KMO (IC 95\%) & Prueba de esfericidad de Bartlett & $\begin{array}{l}\text { Varianza } \\
\text { explicada }\end{array}$ \\
\hline Cohesión & .090 & $.848(.822-.886)$ & $\chi^{2}(45)=1018.2 ; \mathrm{p}<.0001$ & $43.6 \%$ \\
Adaptabilidad & .327 & $.623(.585-.694)$ & $\chi^{2}(45)=472.4 ; \mathrm{p}<.0001$ & $23.8 \%$ \\
\hline
\end{tabular}

Se utilizó el criterio de análisis paralelo (Timmerman \& Lorenzo-Seva, 2011), para extraer y determinar el número de factores más apropiado, coincidiendo en ambos casos en una solución unifactorial. Se utilizó el método de estimación de factores de mínimos cuadrados no ponderados 
(MLS)sin rotación, ya que solo evidencia un factor (Lorenzo-Seva, 1999); se consideran las cargas óptimas mayores a .300 (Hair et al., 1999).

\section{Tabla 4.}

Cargas factoriales y comunalidades para la escala de cohesión y adaptabilidad familiar.

\begin{tabular}{|c|c|c|c|c|c|}
\hline \multicolumn{3}{|c|}{ Cohesión } & \multicolumn{3}{|c|}{ Adaptabilidad } \\
\hline Ítem & $\mathrm{F} 1$ & $h^{2}$ & Ítem & $\mathrm{F} 2$ & $h^{2}$ \\
\hline Ítem 1 & .693 & .480 & Ítem 2 & .422 & .178 \\
\hline Ítem 3* & .197 & .039 & Ítem 4 & .424 & .180 \\
\hline Ítem 5 & .497 & .247 & Ítem $6^{*}$ & .093 & .009 \\
\hline Ítem 7 & .411 & .169 & Ítem 8 & .631 & .398 \\
\hline Ítem 9 & .720 & .519 & Ítem 10 & .429 & .184 \\
\hline Ítem 11 & .754 & .568 & Ítem $12 *$ & .193 & .037 \\
\hline Ítem 13 & .544 & .296 & Ítem $14 *$ & .264 & .070 \\
\hline Ítem 15 & .516 & .266 & Ítem 16 & .587 & .344 \\
\hline Ítem 17 & .595 & .354 & Ítem $18^{*}$ & .060 & .004 \\
\hline Ítem 19 & .521 & .271 & Ítem $20 *$ & .103 & .011 \\
\hline
\end{tabular}

Nota: * items eliminado porque las cargas factoriales no superan el .300

En la dimensión de cohesión, el ítem 3 carece de una carga factorial adecuada por lo que se lo eliminó para análisis posteriores. Asimismo, en la escala de adaptabilidad los ítems 6, 12, 14, 18 y 20 no superaron los niveles de .300 , por lo que también se los eliminó. Se repitió el análisis de KMO $[\mathrm{IC} 95 \%]=.656[.610-.717] ; \chi^{2}(10)=300.4 ; \mathrm{p}<.0001$. Donde el límite 
superior del KMO alcanza un valor aceptable y se continuó con el análisis de la dimensión de 5 ítems.

\section{Análisis Factorial Confirmatorio.}

Se realizó el AFC, con base en modelos con todos los ítems y los modelos con ítems eliminados. Se utilizó el método de estimación de mínimos cuadrados no ponderados debido a que no se cumplió el criterio de normalidad multivariada (Lloret-Segura et al., 2014).

Para interpretar el modelo factorial de manera eficiente se utilizaron varios indicadores de ajuste, permitiendo así una mejor evaluación (Arias, 2008; Ferrando \& Anguiano-Carrasco, 2010; Hair et al., 1999; Hu \& Bentler, 1998; Lloret-Segura et al., 2014; Manzano \& Zamora, 2010; Ruíz, Hernández, Mayrén, \& Vargas, 2014). Los indicadores seleccionados fueron Chicuadrado $\left(X^{2}\right)$, Índice de Bondad de Ajuste $(G F I)$, Índice de Aproximación de la Raíz de Cuadrados Medios del Error (RMSEA), Índice de la Raíz del Cuadrado Medio del Residuo ( $R M R$ ), Índice de Ajuste Comparativo (CFI), Criterio de Información de Akaike $(A I C)$ y la Constante del Criterio de Información de Akaike (CAIC).

El $X^{2}$ mostró un ajuste adecuado, en tanto exista un nivel de significación asociado mayor a .05 , aceptando la hipótesis nula de que todos los errores del modelo son nulos (Ruiz, Pardo \& San Martín, 2010). Así también el $X^{2} / g l$, señaló un buen ajuste debido a que resultó menor a tres (Ruiz et al., 2010).

Los valores del CFI, GFI y $A G F I$, señalan un buen ajuste debido a que superaron el valor de .95 (Hair et al., 1999; Ferrando \& Anguiano-Carrasco, 2010; Manzano \& Zamora, 2010; Ruiz et al., 2010; Lloret-Segura et al., 2014). El RMSEA y el $R M R$, señalan un óptimo ajuste con valores por debajo a .05 (Lloret-Segura et al., 2014).

Los índices de $A I C$ y la CAIC se utilizan para comparar soluciones factoriales e identificar cual es la que presenta un mejor ajuste, con base en el menor valor posible encontrado (Byrne, 1994) (ver Tabla 4). 


\section{Tabla 5.}

Índice de ajuste de las escalas de cohesión y adaptabilidad con todos los ítems y los modificados.

\begin{tabular}{|c|c|c|c|c|c|c|c|c|c|c|}
\hline Modelos & $X^{2}$ & g.l. & $X^{2} / g . l$. & CFI & $G F I$ & $A G F I$ & RMSEA [IC 95\%] & $R M R$ & $A I C$ & CAIC \\
\hline Cohesión & $72.055^{*}$ & 34 & 2.12 & .955 & .982 & .970 & $.054[.049-.095]$ & .069 & 4.05 & -144.38 \\
\hline Cohesión ${ }^{\text {a }}$ & $55.075^{*}$ & 26 & 2.12 & .984 & .987 & .978 & $.036[.045-.099]$ & .059 & 3.07 & -110.44 \\
\hline Adaptabilidad & $98.241^{*}$ & 34 & 2.89 & .588 & .947 & .915 & $.105[.072-.116]$ & .098 & 30.24 & -118.20 \\
\hline Adaptabilidad $^{\text {b }}$ & $24.405^{*}$ & 4 & 6.10 & .879 & .982 & .933 & $.141[.099-.216]$ & .081 & 16.40 & -1.06 \\
\hline
\end{tabular}

Nota: ${ }^{*} p<0.001 ;{ }^{a}=$ versión de la escala de cohesión familiar con el ítem 3 eliminado; ${ }^{b}=$ versión de la escala de adaptabilidad con el item 6, 12, 14, 18 y 20

La versión ambas versiones de cohesión presentan CFI, GFI, AGFI, RMSEA, RMR adecuados, el CAIC es mucho menor en la versión completa, por lo que, si bien ambos modelos tienen niveles adecuados, se opta por analizar la versión de cohesión completa para no perder información que aporta el reactivo $\mathrm{N}^{\circ} 3$.

El mejor ajuste se da para la escala de cohesión con todos los ítems, siendo apoyado por los resultados del AFE. Asimismo, en la escala de adaptabilidad familiar no presentan evidencia de una adecuada estructura interna, tanto en la versión con todos los ítems como en la versión con ítems eliminados. Por lo que se decide realizar los análisis solo con la escala de cohesión familiar ya que presenta una estructura interna adecuada.

\section{Análisis de confiabilidad.}

Finalmente se analizó la confiabilidad de la escala de cohesión familiar con todos los ítems mediante el estadístico de $\alpha$ de Cronbach .789 [IC 95\% $=.758$ -.818 y y $\omega=.81$. Considerándose adecuadas en ambos casos (Dominguez- 
Lara \& Merino, 2015; Koning \& Franses, 2003). Todos los ítems presentaban una correlación ítems-test mayor a .20 (DeVellis, 2012).

\section{Diferencias entre el tipo de familia.}

Posteriormente, se analizó la existencia de diferencias significativas entre los tipos de composición familiar (extensa, nuclear, monoparental y anuclear) y la cohesión familiar (tabla 6). Se encontró que existen diferencias significativas entre los puntajes de cohesión en adolescentes provenientes de familias anucleares $(n=36,8.4 \%)$ y familias extensas $(n=166,38.8 \%)$, así como de familias anucleares $(n=36,8.4 \%)$ y nucleares $(n=170,39.7 \%)$.

\section{Tabla 6.}

Análisis de los cuatro tipos de composición familiar según la cohesión familiar $(n=428)$.

\begin{tabular}{cccccc}
\hline & Grupo 1 & Grupo 2 & $h$ & $E E$ & $p$ \\
\hline Cohesión & Anuclear & Monoparental & 55.635 & 26.382 & .035 \\
& Anuclear & Extensa & 85.416 & 22.705 & .000 \\
& Anuclear & Nuclear & 96.601 & 22.658 & .000 \\
& Monoparental & Extensa & -29.781 & 19.085 & .119 \\
& Monoparental & Nuclear & 40.966 & 19.028 & .031 \\
& Extensa & Nuclear & 11.185 & 13.476 & .407 \\
\hline
\end{tabular}

Nota: $h=$ valor del estadístico $h$ de Kruskal Wallis; $E E=$ error estándar

Al analizar el rango promedio de los puntajes de cohesión, y comparándolo con los tipos de composición familiar, se aprecia una diferencia significativa entre los puntajes de adolescentes pertenecientes a familias anuclearesnucleares y anucleares-extensas, tanto en las dimensiones de cohesión y adaptabilidad. 


\section{Cohesión.}

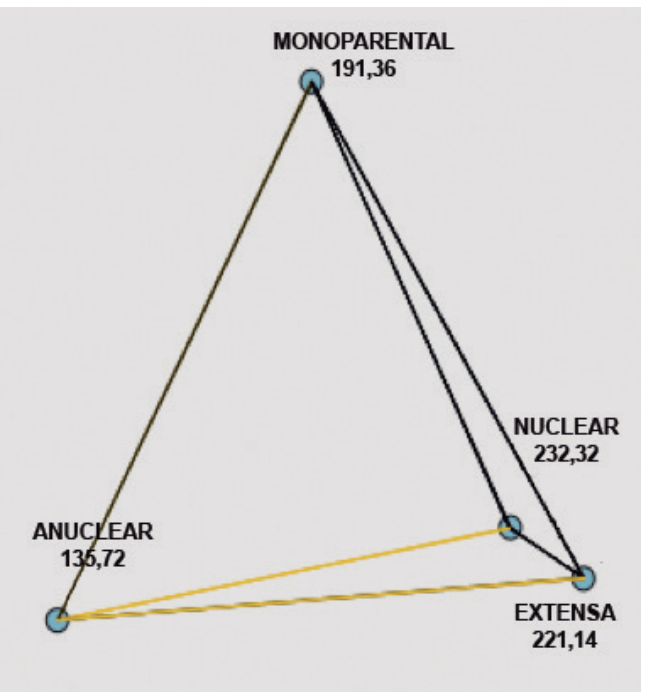

Figura 1. Comparación por parejas del rango promedio de los puntajes de cohesión según el tipo de composición familiar.

Si bien los datos evidencian una diferencia estadísticamente significativa, el tamaño muestral de los grupos es variado (entre 36 a 170), esto podría ser una fuente de error, ya que el estadístico $h$ de Kruskal Wallis utiliza como insumo el tamaño de los grupos (Shapiro \& Wilk,1965; Ghasemi, \& Zahediasl, 2012). De manera que se homogenizaron los grupos, seleccionando 36 adolescentes de cada tipo de composición familiar de forma aleatoria. El análisis de estos grupos mostró que se mantienen las diferencias significativas entre las familias nucleares y anucleares solo en la dimensión de cohesión familiar (tabla 7) (figura 2). 


\section{Tabla 7.}

Análisis de los cuatro tipos de composición familiar según la cohesión $(n=144)$.

\begin{tabular}{lllll}
\hline & Grupo 1 & Grupo 2 & $h$ & $p$ \\
\hline Cohesión & Anuclear & Monoparental & 20.792 & .034 \\
& Anuclear & Extensa & 22.931 & .019 \\
& Anuclear & Nuclear & 29.500 & .003 \\
& Monoparental & Extensa & -2.139 & .827 \\
& Monoparental & Nuclear & 8.708 & .375 \\
& Extensa & Nuclear & 6.569 & .503 \\
\hline
\end{tabular}

Nota: $h=$ valor del estadístico $h$ de Kruskal Wallis; $E E=9.814$

\section{Cohesión.}

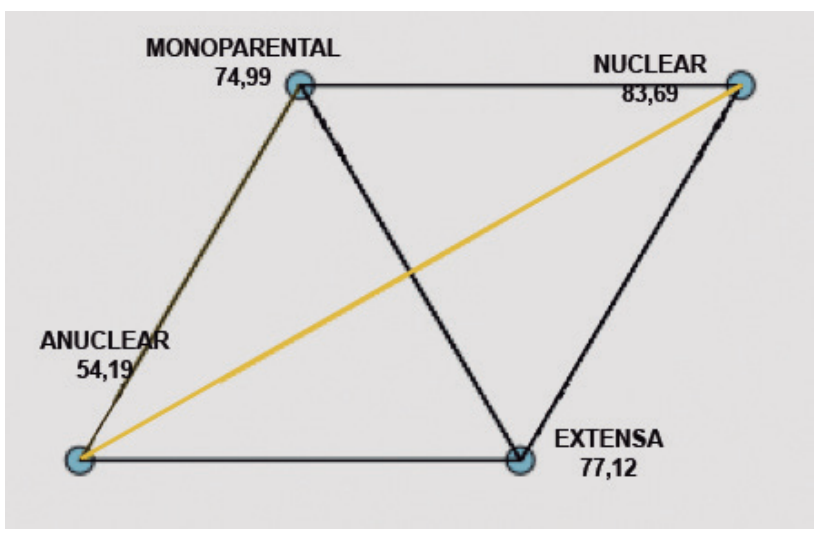

Figura 2. Comparación por parejas del rango promedio de los puntajes de cohesión según el tipo de composición familiar.

Asimismo, pese al reducido tamaño de muestra, existen diferencias significativas entre la percepción de la cercanía emocional (cohesión) entre los miembros de la familia, de adolescentes pertenecientes a familias que viven con ambos padres (nucleares) y familias donde el apoderado no es el 
padre/madre biológico(a) o legal, sino otro miembro como un tío, hermano mayor o abuelo que cumplen las funciones parentales (familia anuclear).

\section{Discusión}

Este estudio buscó determinar la existencia de diferencias entre la cohesión y adaptabilidad familiar, según la composición de los miembros de la familia en un grupo de adolescentes escolares de la provincia constitucional del Callao. Se utilizó la escala FACES III diseñada por Olson et al. (1982) y una ficha técnica para describir la tipología familiar (anuclear, nuclear, monoparental).

El análisis de las relaciones familiares según el tipo de composición evidenció que existen diferencias significativas $(n=428)$ en la cercanía emocional de los adolescentes pertenecientes a familias nucleares y anucleares $(h=85.416 ; p<.001)$, así como de adolescentes pertenecientes a familias extensas y anucleares $(h=96.601 ; p<.001)$. Sin embargo, los grupos (tipos de familia) presentaron tamaños muestrales muy diferentes, por lo que se optó por reducir la muestra en grupos homogéneos ( $n=36$, cada uno); al hacerlo, se encontró que se mantenían las diferencias significativas entre en la cercanía emocional solo, en los adolescentes pertenecientes a familias nucleares y anucleares $(h=29.500 ; p=.003)$, siendo mayor en las familias nucleares.

Estos resultados sugieren que la presencia de al menos uno de los padres biológicos o legales, se encontraría relacionado con mayores niveles de vinculación afectiva percibida por los adolescentes. Así mismo, y en coherencia con Luengo (2008), Sobrino (2008), Bardales (2005), este estudio permitiría concluir que la composición familiar influye en las interacciones familiares, especialmente en la vinculación emocional entre los miembros.

Esto podría deberse a que la ausencia de una figura parental estable, genera la percepción en el adolescente de escasa cercanía emocional con el resto del sistema familiar. Por lo que sería menester que los miembros de las familias anucleares puedan suplir de forma conjunta o mediante un 
responsable determinado, que los adolescentes puedan vincularse de forma más cercana, íntima y con mayor confianza con el resto de los miembros de la familia (hermanos, abuelos, tíos, primos, etc).

Por otra parte, el análisis de estructura interna del FACES III mediante el AFE muestra una adecuación muestral óptima (KMO > .70; Pardo \& Ruiz, 2002; Hair et al., 1999), y un adecuado porcentaje de varianza explicada ( $>$ 40\%; Lloret-Segura et. al., 2014), únicamente en la dimensión de cohesión. Sin embargo, uno de sus ítems no cumplió los valores mínimos dentro de las cargas factoriales (>.300; Hair et al., 1999) por lo que se decidió realizar un AFC con las subescalas originales de cohesión (todos los ítems) y las subescalas modificadas (con un ítem eliminado). Este análisis determinó que la subescala de cohesión original presentaba una mejor solución factorial en comparación a la versión modificada, empero; la subescala de adaptabilidad familiar presentaba propiedades psicométricas inadecuadas, de manera que se la excluyó de análisis posteriores. Con base en los resultados, los hallazgos muestran que el FACES III carece de una adecuada solución factorial, en contraposición a lo propuesto originalmente por Olson et al. (1982), y se observa una inconsistencia factorial sobre la dimensión de adaptabilidad familiar al igual que en estudios previos (Kouneski, 2002; Mirnics, Vargha, Tóth \& Bagby, 2010; Schmidt, Barreyro \& Maglio, 2010).

Entre las limitaciones del estudio, se encuentran las referidas al proceso de muestreo (no probabilística); de manera que las conclusiones se remiten a la muestra estudiada. Además las dificultades respecto al instrumento FACES III, impidieron cumplir el objetivo de evaluar la adaptabilidad familiar; esto debido a que la escala reportó, en este estudio, propiedades psicométricas inadecuadas.

De igual manera, la complejidad de las relaciones familiares, exigen que las investigaciones futuras incluyan variables como la influencia de la disciplina familiar, la comunicación entre los miembros de la familia, el ejercicio de los roles parentales, entre otros; esto permitiría una mejor comprensión de la dinámica familiar en cada contexto. 
Finalmente se concluye que a) existen diferencias significativas entre la vinculación emocional entre los miembros de la familia (cohesión) percibida por lo adolescentes entre familias anucleares y nucleares; por otra parte b) el FACES III presenta inadecuadas propiedades psicométricas a nivel global, por lo que se recomienda mayor estudio al respecto y análisis más robustos. Se espera que los hallazgos de este estudio sirvan para investigaciones posteriores en nuestro contexto.

\section{Referencias}

Arias, B. (2008). Desarrollo de un ejemplo de análisis factorial confirmatorio con LISREL, AMOS y SAS. Trabajo presentado en Seminario de Actualización en Investigación sobre Discapacidad (SAID). Recuperado de: http://gredos.usal.es/jspui/bitstream/10366/82465/1/ VI_SimposioCientificoSaid.pdf\#page $=76$

Ato, M., López, J., \& Benavente, A. (2013). Un sistema de clasificación de los diseños de investigación en psicología. Anales en Psicología, 29(3), 1038-1059. https://doi.org/10.6018/analesps.29.3.178511

Bardales, M. (2005). Organización y funcionalidad familiar y su relación con los niveles de inestabilidad emocional en menores de 6 a 12 años que asisten a una institución de salud mental (Tesis Doctoral). Universidad Inca Garcilaso de la Vega, Lima.

Bermúdez, C., \& Brik, E. (2010). Terapia familiar sistémica. Barcelona: Editorial Síntesis.

Byrne, B. M. (1994). Structural equation modeling with EQS and EQS/ Windows. Thousand Oaks, CA: Sage Publications.

Cervini, R., Dari, N., \& Quiroz, S. (2016). Estructura familiar, tamaño de la familia y el rendimiento en matemática y lectura: análisis comparativo entre países de América Latina. Perfiles Educativos, 38(151), 12-31.

Child Trends (2013). Mapa mundial de la familia: Los cambios en la familia y su impacto en el bienestar de la niñez. Traducido por el Instituto de Ciencias para la Familia de la Universidad Nacional de Piura, Lima. 
Copez-Lonzoy, A., Villarreal-Zegarra, D., \& Paz-Jesús, A. (2016). Propiedades psicométricas de la Escala de Comunicación Familiar en estudiantes universitarios. Revista Costarricense de Psicología, 35(1), $37-52$.

DeVellis, R. F. (2012). Scale Development, Theory and Applicatons. Los Angeles: SAGE.

Dominguez-Lara, S. (2016). Valores normativos de una escala de autoeficacia académica en estudiantes universitarios de Lima. Interacciones, 2(2), 91-98. https://doi.org/10.24016/2016.v2n2.31

Domínguez-Lara, S., \& Merino, C. (2015). ¿ Por qué es importante reportar los intervalos de confianza del coeficiente alfa de Cronbach?. Revista Latinoamericana de Ciencias Sociales, Niñez y Juventud, 13(2), 13261328.

Faúndes, J. M. (2012). Sexualidad y alteridad: argumentos del conservadurismo religioso cordobés contra el matrimonio" igualitario". Sociedade e Cultura, 15(2), 347-358

Ferrando, P. J., \& Anguiano-Carrasco, C. (2010). El análisis factorial como técnica de investigación en psicología. Papeles del Psicólogo, 31(1), 1833.

Ghasemi, A., \& Zahediasl, S. (2012). Normality Tests for Statistical Analysis: A Guide for Non-Statisticians. International Journal of Endocrinology and Metabolism, 10(2), 486-489. https://doi.org/10.5812/ijem.3505

Hair, J.F.; Anderson, R.E.; Tatham, R.L., \& Black, W. (1999). Análisis Multivariante. Madrid: Prentice Hall.

Hu, L., \& Bentler, P. (1998). Fit indices in covariance structure modeling: Sensivity to underparameterized model misspecification. Psychological Methods, 3(4), 424-453. https://doi.org/10.1037/1082-989X.3.4.424

Kouneski, E. (2002). Circumplex model and FACES: Review of literature. Recuperado de: http://www.facesiv.com.

Lloret-Segura, S., Ferreres-Traver, A., Hernández-Baeza, A., \& TomásMarco, I. (2014). El análisis factorial exploratorio de los Ítems: una guía práctica, revisada y actualizada. Anales de Psicología, 30(3), 1151-1169. https://doi.org/10.6018/analesps.30.3.199361 
López-Monta-o, L. M., \& Herrera-Saray, G. D. (2014). Epistemología de la ciencia de familia-Estudios de familia. Revista Latinoamericana de Ciencias Sociales, Niñez y Juventud, 12(1), 65-76. https://doi.org/10.11 600/1692715x.1212080413

Lorenzo-Seva, U. (1999). Promin: A method for oblique factor rotation. Multivariate Behavioral Research, 34, 347-365.https://doi.org/10.1207/ S15327906MBR3403_3

Luengo, T. (2008). Un análisis de la nuclearidad parsoniana a partir de una investigación sobre la relación entre estructura familiar y satisfacción parental. Lima. Revista de Investigación en Psicología UNMSM, 11(2), $13-27$

Manzano, A., \& Zamora, S. (2010). Sistema de ecuaciones estructurales: una herramienta de investigación. México D.F.: Centro Nacional de Evaluación para la Educación Superior.

Martínez, S. D. (2010). La Educación, cosa de dos: La escuela y la familia. Revista Digital para Profesionales de la Enseñanza, 1(8), 1-15.

Mirnics, Z., Vargha, A., Tóth, M., \& Bagdy, E. (2010). Cross-Cultural Applicability of FACES IV. Journal of Family Psychotherapy, 21(1), 17-33. https://doi.org/10.1080/08975351003618577

Pardo, A., \& Ruiz, M. (2002). SPSS 11: Guía para el análisis de datos. España: McGrawHill

Olson, D., McCubbin, H., Barnes, H., Larsen, A., Muxen, M., \& Wilson, M. (1982). Family Inventories. Inventories used in a national survey of family across the family life cicle. Family Social Science. University of Minnesota.

Olson, D. H. (2000). Circumplex Model of Marital and Family Systems. Journal of Family Therapy, 22(2), 144-167.https://doi.org/10.1111/14676427.00144

Olson, D. (2011). Faces IV and the circumplex model: Validation study. Journal of Marital and Family Therapy, 37(1), 64-80. https://doi. org/10.1111/j.1752-0606.2009.00175.x 
Orcasita, L. T., \& Uribe, A. F. (2010). La importancia del apoyo social en el bienestar de los adolescentes. Psychologia. Avances de la Disciplina, 4(2), 69-82.

Reusche, R. M. (1998). El funcionamiento familiar de adolescentes con problemas de conducta escolar, según la Escala para evaluar la Adaptabilidad y Cohesión de la familia (FACES III) de D.H. Olson. (Tesis Doctoral). Universidad Femenina del Sagrado Corazón, Lima.

Ruíz, A., Hernández, M., Mayrén, P., \& Vargas M. (2014). Funcionamiento familiar de consumidores de sustancias adictivas con y sin conducta delictiva. Liberabit, 20(1), 109-119.

Ruiz, M., Pardo, A., \& San Martín, R. (2010). Modelos de Ecuaciones Estructurales. Papeles del Psicólogo, 31(1), 34-45.

Scarpati, M. P., Pertuz, M. S., \& Silva, A. S. (2014). Límites, reglas, comunicación en familia monoparental Con hijos adolescentes. Diversitas: Perspectivas en Psicología, 10(2), 225-246. https://doi. org/10.15332/s1794-9998.2014.0002.03

Shapiro, S.S., \& Wilk, M.B. (1965). An Analysis of Variance Test for Normality (complete Samples). Biometrika, 52(3/4), 591-611. https:// doi.org/10.1093/biomet/52.3-4.591

Schmidt, V., Barreyro, J. P., \& Maglio, A. L. (2010). Escala de evaluación del funcionamiento familiar FACES III: ¿ Modelo de dos o tres factores?. Escritos de Psicología, 3(2), 30-36.

Sobrino, L. (2008). Niveles de satisfacción familiar y de comunicación entre padres e hijos. Avances en Psicología, 16(1). 109-137.

Staccini, L., Tomba, E., Grandi, S., \& Keitner, G. I. (2015). The evaluation of family functioning by the family assessment device: A systematic review of studies in adult clinical populations. Family Process, 54(1), 94-115. https://doi.org/10.1111/famp.12098

Suárez, M., Díaz Bringas, M., Sánchez Lozada, M., Fernández Cárdenas, E. J., Carrillo Alfonso, T., \& Guasch Almeida, T. (2015). Caracterización de la salud familiar en estudiantes de Medicina. Revista Médica Electrónica, 37(2), 121-129. 
Timmerman, M. E., \& Lorenzo-Seva, U. (2011). Dimensionality Assessment of Ordered Polytomous Items with Parallel Analysis. Psychological Methods, 16, 209-220. https://doi.org/10.1037/a0023353

Toscano, F. (1998). Caracteristicas disfuncionales en familias con hijos varones dependientes de sustancias psicoactivas institucionalizados en hospitales de rehabilitación de Lima (Tesis de Licenciatura). Universidad Inca Garcilaso de la Vega, Lima. 